\title{
Adropin reduces hypoxia/reoxygenation-induced myocardial injury via the reperfusion injury salvage kinase pathway
}

\author{
LINGZHEN WU, JUN FANG, XUN YUAN, CHANG XIONG and LIANGLONG CHEN \\ Department of Cardiology, Fujian Medical University Union Hospital, Fuzhou, Fujian 350001, P.R. China
}

Received October 14, 2018; Accepted May 10, 2019

DOI: $10.3892 / \mathrm{etm} .2019 .7937$

\begin{abstract}
Adropin is a secreted polypeptide that has been demonstrated to serve an important role in protecting the vascular endothelium. Pharmacological activation of pro-survival kinases, such as PI3K-Akt and ERK1/2, are involved in the reperfusion injury salvage kinase (RISK) pathway. In the present study, the effects of adropin in cardiomyocyte injury induced by simulated ischemia/reperfusion (SI/R) were assessed. Additionally, the current study also assessed the mechanisms that govern SI/R in a H9c2 cardiomyoblast cell model. Cell viability was measured using an MTT assay. Cell injury was assessed using creatine kinase MB measurements. Apoptosis was assessed using flow cytometry and caspase-3 activity. The inflammatory response was measured using tumor necrosis factor $\alpha$ and interleukin-10 expression. Oxidative stress was assessed using malondialdehyde and superoxide dismutase. The expression levels of Akt, ERK1/2, glycogen synthase kinase $3 \beta$ (GSK3 $\beta$ ), Bcl-2 and Bax were determined using western blot analysis. The results of the current study revealed that moderate-dose adropin increased cell viability, reduced early apoptosis and caspase-3 activity, promoted Bcl-2 expression, inhibited Bax and increased the Bcl-2/Bax ratio. Adropin significantly increased the phosphorylation of Akt, ERK1/2 and GSK3 $\beta$, whereas inhibitors of PI3K and ERK1/2, respectively, LY294002 and PD98059, abolished the cardioprotective role of adropin. Furthermore, no significant difference was observed in phosphorylated-STAT3/total-STAT3 expression between the adropin and SI/R groups and Janus kinase 2 inhibitor AG490 did not significantly inhibit the protective role of adropin. These results indicate that adropin exerts a protective effect against SI/R injury through the RISK pathway instead of the survivor activating factor enhancement pathway.
\end{abstract}

Correspondence to: Dr Lianglong Chen or Dr Jun Fang, Department of Cardiology, Fujian Medical University Union Hospital, 29 Xin-Quan Road, Fuzhou, Fujian 350001, P.R. China E-mail: lianglongchenxh@126.com

E-mail: ptfangjun@126.com

Key words: myocardial reperfusion injury, postconditioning, adropin, reperfusion injury salvage kinase pathway

\section{Introduction}

The most effective strategy to reduce acute myocardial ischemic injury and subsequent mortality is to promptly recover coronary reflow using thrombolytic therapy or percutaneous intervention (1). However, reperfusion can induce myocardial ischemia reperfusion injury (MIRI). The inflammatory response, oxidative stress and cell apoptosis are considered to be critical factors associated with mediating the effects of MIRI (2-4). Targeting these factors is important in the prevention and reduction of MIRI.

Adropin, first described by Kumar et al (5) in 2008, is a secreted protein and an endogenous biologically active substance encoded for by an energy homeostasis-associated gene. Lovren et al (6) demonstrated that adropin is expressed in the endothelial cells of the umbilical veins and coronary arteries. The aforementioned study also revealed that adropin may exhibit nonmetabolic properties, which includes the regulation of endothelial function through the upregulation of endothelial nitric oxide synthase (eNOS) via the PI3K-Akt and ERK1/2, which are the two major components of the reperfusion injury salvage kinase (RISK) pathway. The RISK pathway represents one of the most important survival mechanisms against ischemic reperfusion injury. Apart from the RISK pathway, the survivor activating factor enhancement (SAFE) pathway also serves a role in ischemic postconditioning. The major components of the SAFE pathway are TNF- $\alpha$ and Janus kinase (JAK), which phosphorylates the transcription factor STAT3. Additionally, adropin has been revealed to improve murine limb perfusion and elevate capillary density following the induction of hindlimb ischemia (6). Clinical research has demonstrated that adropin is associated with a variety of metabolic risk factors. Butler et al (7) demonstrated that plasma adropin levels are negatively associated with obesity and insulin resistance. Celik et al (8) revealed that serum adropin levels were negatively associated with cardiac X syndrome due to coronary microvascular perfusion dysfunction and that low serum adropin levels were an independent risk factor of $\mathrm{X}$ syndrome. Adropin has been revealed to be negatively correlated with inflammatory biomarker-C reactive protein and it has been demonstrated that patients with severe atherosclerosis exhibit lower adropin levels (9). These results indicated that adropin may influence the anti-inflammatory response and reduce atherosclerosis (9). Yang et al (10) demonstrated that adropin reduces endothelial cell permeability and modulates 
ischemia-induced blood-brain barrier injury. However, to the best of our knowledge, the role of adropin in myocardial reperfusion injury has not yet been assessed.

In the current study, a hypoxia/reoxygenation model was established in neonatal rat cardiomyoblast cells (H9c2) to simulate ischemia/reperfusion (SI/R) injury. The effect of adropin on SI/R injury and the mechanisms that govern this effect were subsequently assessed.

\section{Materials and methods}

Cell culture. H9c2 cells were obtained from the Type Culture Collection of the Chinese Academy of Sciences. Cells were passaged up to 4 times and were cultured in DMEM (GE Healthcare Life Sciences) containing 10\% (v/v) heat-inactivated FBS (Gibco; Thermo Fisher Scientific, Inc.), 100 IU/ml penicillin (GE Healthcare Life Sciences) and $100 \mu \mathrm{g} / \mathrm{ml}$ streptomycin (GE Healthcare Life Sciences), under a $5 \% \mathrm{CO}_{2}$ atmosphere at $37^{\circ} \mathrm{C}$.

H9c2 cells subjected to hypoxia/reoxygenation induced injury. Hypoxia was induced as described previously (11). H9c2 cells were cultured to $70-80 \%$ confluency, fresh DMEM without FBS was subsequently added and the cells were transferred to a triple gas incubator with either hypoxic $\left(5 \% \mathrm{CO}_{2}, 1 \% \mathrm{O}_{2}\right.$ and $94 \% \mathrm{~N}_{2}$ ) or SI/R (hypoxia: $5 \% \mathrm{CO}_{2}, 1 \% \mathrm{O}_{2}$ and $94 \% \mathrm{~N}_{2}$, followed by reoxygenation: $5 \% \mathrm{CO}_{2}, 21 \% \mathrm{O}_{2}$ and $74 \% \mathrm{~N}_{2}$ ) settings. A hypoxia/reoxygenation model was established and cells were divided into 11 groups. All groups except the control group were treated with hypoxic conditions for $12 \mathrm{~h}$ and reoxygenation for $24 \mathrm{~h}$. Postconditioning of cardiomyocytes was achieved as follows: At the end of $12 \mathrm{~h}$ of hypoxia, the cells were initially received different doses of adropin and then returned to the reoxygenated condition for another $24 \mathrm{~h}$. The groups were classified as follows: i) Control group, normoxic conditions $\left(37^{\circ} \mathrm{C}, 5 \% \mathrm{CO}_{2}, 21 \% \mathrm{O}_{2}, 71 \% \mathrm{~N}_{2}\right)$; ii) $\mathrm{SI} / \mathrm{R}$ group; iii) SI/R + low dose adropin (10 ng/ml; Phoenix Pharmaceuticals, Inc.), in which adropin was added prior to reoxygenation (adropin-L); iv) SI/R + moderate dose adropin group (25 ng/ml; adropin-M); v) SI/R + high dose adropin group (50 ng/ml; adropin-H); vi) LY294002 group, $40 \mu \mathrm{mol} / 1$ PI3K specific inhibitor LY294002 (Sigma-Aldrich; Merck $\mathrm{KGaA}$ ) was added to the medium prior to hypoxia as described previously (12); vii) adropin + LY294002 group, in which $40 \mu \mathrm{mol} / 1 \mathrm{LY} 294002$ and $25 \mathrm{ng} / \mathrm{ml}$ adropin were added to the medium prior to hypoxia (12) and reoxygenation, respectively; viii) PD98059 group, in which $25 \mu \mathrm{mol} / 1$ ERK1/2-specific inhibitor PD98059 (Sigma-Aldrich; Merck KGaA) was added to the medium (12) prior to hypoxia; ix) adropin + PD98059 group, in which $25 \mu \mathrm{mol} / 1 \mathrm{PD} 98059$ and $25 \mathrm{ng} / \mathrm{ml}$ adropin were added to the medium (12) prior to hypoxia and reoxygenation, respectively; x) AG490 group, in which $100 \mu \mathrm{mol} / 1 \mathrm{JAK} 2$ inhibitor AG490 (Sigma-Aldrich; Merck KGaA) was added to the medium prior to hypoxia as described previously (13); xi) adropin + AG490 group, in which $100 \mu \mathrm{mol} / 1 \mathrm{AG} 490$ and $25 \mathrm{ng} / \mathrm{ml}$ adropin were added to the medium (13) prior to hypoxia and reoxygenation, respectively.

MTT measurement of cell viability. A total of $1 \times 10^{5} \mathrm{H} 9 \mathrm{c} 2$ cells $/ \mathrm{ml}$ were seeded into a 96 -well culture plate and incubated at $5 \% \mathrm{CO}_{2}$ and $37^{\circ} \mathrm{C}$ for $24 \mathrm{~h}$. Cell viability was determined using an MTT assay. At $12 \mathrm{~h}$ following reoxygenation, $20 \mu \mathrm{l}$ MTT solution was added into each well $(5 \mathrm{mg} / \mathrm{ml})$ and plates were incubated for $4 \mathrm{~h}$ at $37^{\circ} \mathrm{C}$. A microplate reader was used to measure the absorbance at a wavelength of $490 \mathrm{~nm}$.

ELISA assay and colorimetry. The expression of creatine kinase MB (CK-MB; cat. no. H197), tumor necrosis factor $\alpha$ (TNF- $\alpha$; cat. no. H052) and interleukin (IL)-10 (cat. no. H009) were measured using ELISA assay kits (Nanjing Jiancheng Bioengineering Institute). Malondialdehyde (MDA; cat. no. A003-4) and superoxide dismutase (SOD; cat. no. A001-1) concentrations were determined using colorimetry kits according to manufacturer's protocols (Nanjing Jiancheng Bioengineering Institute). The experiment was performed at least three times and CK-MB level was expressed as IU/1. TNF- $\alpha$ and IL-10 levels were expressed as pg/ml. The MDA level and SOD were expressed as $\mathrm{nmol} / \mathrm{mg}$ protein and as $\mathrm{U} / \mathrm{mg}$ protein, respectively.

Apoptosis analysis. Early cell apoptosis was measured using flow cytometry. The analysis of phosphatidylserine on the outer apoptotic cell membranes was performed using annexin-Vfluorescein and propidium iodide (Annexin-V-FLUOS Staining kit; Roche Diagnostics). Collected cells were rinsed with ice-cold PBS and resuspended in $250 \mu \mathrm{l}$ of binding buffer and $\sim 1-5 \times 10^{5}$ cells were analyzed in each of the samples. A total of $100 \mu \mathrm{l}$ annexin-V-FLUOS labeling solution was added to the cells, which were then incubated for $15 \mathrm{~min}$ at $25^{\circ} \mathrm{C}$. The cells were analyzed using FlowJo software (version 10.4.1; BD FACScanto II; Becton, Dickinson and Company).

Measurement of caspase-3 activity. Caspase-3 activity was measured using a colorimetric activity assay kit (Ac-DEVD-pNA; Beyotime Institute of Biotechnology) according to manufacturer's protocol. In brief, cells were lysed in ice-cold lysis buffer, placed on ice for $15 \mathrm{~min}$, then centrifuged at $4^{\circ} \mathrm{C}$ for $15 \mathrm{~min}$ at $16,000 \mathrm{x}$ g and supernatant was subsequently incubated with caspase- 3 substrate on a 96-well plate. Protein concentration was determined using Bradford protein assay kit (cat. no. P0006; Beyotime Institute of Biotechnology). Caspase-3 activity was determined using a microplate reader at a wavelength of $405 \mathrm{~nm}$.

Western blot analysis. H9c2 cells were washed with PBS, enzymatically dissociated with the use of trypsin (HyClone; GE Healthcare Life Sciences), and prepared in lysis buffer with protease inhibitor cocktail (cat. no. P0013B; Beyotime Institute of Biotechnology). Protein quantification was measured by using a BCA protein assay kit (cat. no. P0012; Beyotime Institute of Biotechnology). Equal quantities of protein $(30 \mu \mathrm{g} /$ lane) from whole cell lysates of cultured $\mathrm{H} 9 \mathrm{c} 2$ cells were separated by $10 \%$ SDS-PAGE and transferred to a PVDF membrane. Following blocking with 5\% BSA for $1 \mathrm{~h}$ at room temperature for binding non-specific sites, membranes were incubated with primary antibodies overnight at $4^{\circ} \mathrm{C}$. The following primary antibodies were used: Phosphorylated (p)-Akt polyclonal antibody (1:1,000; cat. no. YP0864) and Akt polyclonal antibody (1:1,000; cat. no. YT0173) were purchased from ImmunoWay Biotechnology Company. 

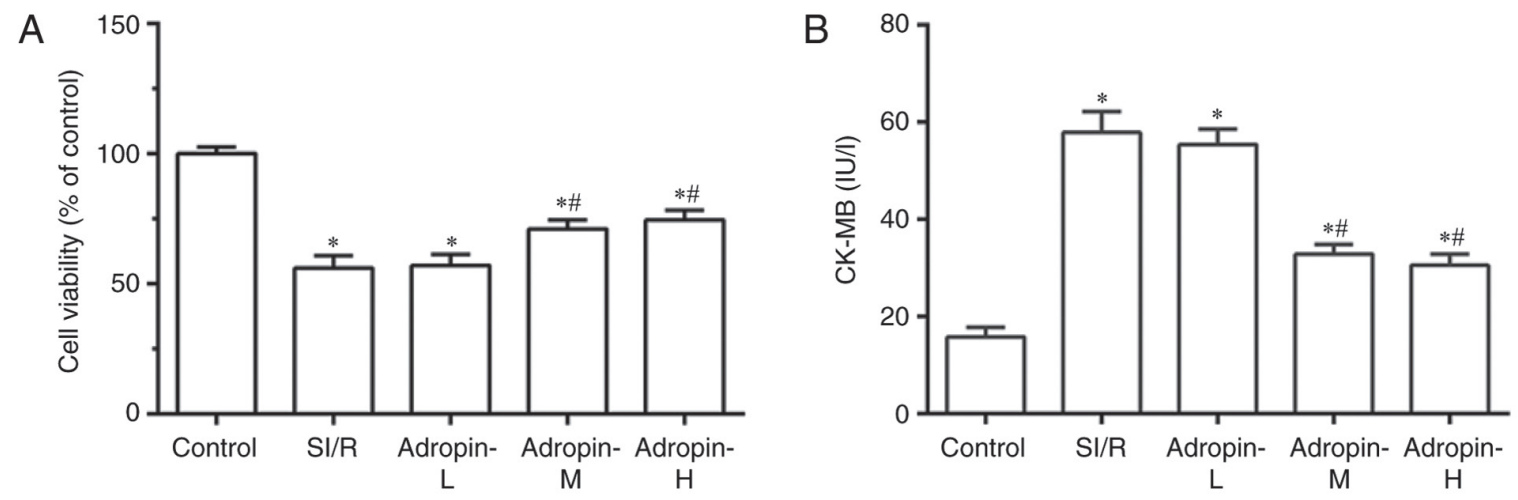

Figure 1. Effect of adropin on cell viability. (A) Cell viability and (B) CK-MB of H9c2 cells subjected to SI/R. " $\mathrm{P}<0.001$ vs. control and ${ }^{\# P} \mathrm{P}<0.001$ vs. SI/R Results are representative of three independent experiments. CK-MB, creatine kinase MB; SI/R, simulated ischemia/reperfusion; L, low; M, medium; H, high .

p-ERK 1/2 monoclonal antibody (1:1,000; cat. no. sc-136521), ERK 1/2 monoclonal antibody (1:1,000; cat. no. sc-514302), p-STAT3 antibody (1:1000; cat. no. sc-7993) and STAT3 antibody $(1: 1,000$; cat. no. sc-8019) were purchased from Santa Cruz Biotechnology, Inc. P-GSK3 $\beta$ antibody (1:1,000; cat. no. ab131097), GSK3 $\beta$ antibody (1:5,000; cat. no. ab32391), Bcl-2 antibody (1:1,000; cat. no. ab59348) and Bax antibody (1:1,000; cat. no. ab32503) were purchased from Abcam. Following incubation with horseradish peroxidase (HRP)-conjugated goat anti-rabbit IgG (1:5,000; cat. no. ZB-5301; OriGene Technologies, Inc.) or HRP-conjugated goat anti-mouse IgG (1:5,000; cat. no. ZB-2305; OriGene Technologies, Inc.) at $37^{\circ} \mathrm{C}$ for $1 \mathrm{~h}$, the signals were detected with Pierce ${ }^{\mathrm{TM}}$ ECL Western Blotting Substrate kit (cat. no. 32209, Pierce; Thermo Fisher Scientific, Inc.) and bands were subsequently quantified using Quantity One software (version 4.6.2; Bio-Rad Laboratories, Inc.).

Statistical analysis. Data are presented as the mean \pm standard deviation. Comparisons between groups were performed using one-way ANOVA with Student-Newman-Keuls correction for multiple comparisons. Statistical analyses were performed using SPSS version 13.0 (SPSS Inc.). $\mathrm{P}<0.05$ was considered to indicate a statistically significant difference.

\section{Results}

Effect of adropin dose on cell viability and CK-MB levels. Cell viability was examined using a MTT assay and CK-MB levels were measured to assess cardiomyocyte injury. As presented in Fig. 1A and B, SI/R group cell viability was significantly reduced $(\mathrm{P}<0.001)$ and $\mathrm{CK}-\mathrm{MB}$ levels significantly increased $(\mathrm{P}<0.001)$ compared with the control group. Cell viability was significantly higher and CK-MB levels were significantly lower in the adropin-M and adropin-H groups when compared with the SI/R group. In addition, no significant difference in cell viability and CK-MB levels was observed between the adropin-M and adropin-H groups. The adropin-L group did not exhibit any significant effect on cell viability or CK-MB expression when compared with the SI/R group, indicating that moderate and high adropin levels can reduce SI/R injury. The subsequent experiments were performed using moderate-dose adropin as the adropin group.
Effect of adropin on myocardial apoptosis. Flow cytometry was used to assess myocardial apoptosis and caspase-3 activity subsequent to reoxygenation (Fig. 2). The SI/R group exhibited a higher early apoptosis rate $(\mathrm{P}<0.001)$ and higher caspase-3 activity $(\mathrm{P}<0.001)$ when compared with the control group (Fig. 2B and C, respectively). Moderate-dose adropin exhibited a significantly lower early apoptosis rate $(\mathrm{P}<0.001)$ and caspase-3 activity $(\mathrm{P}<0.001)$ compared with the $S I / R$ group. Additionally, LY294002 and PD98059 significantly reversed the protective effects of adropin on apoptosis rate $(\mathrm{P}<0.001)$ and significantly increased caspase-3 activity $(\mathrm{P}<0.001)$ compared with the adropin group (Fig. 2A-C). However, AG490 exhibited no significant effect on early apoptosis rate or caspase-3 activity when compared with the adropin group (Fig. 2A-C).

Western blot analysis was used to detect the effect of adropin and the aforementioned inhibitors on the Bcl-2/Bax ratio. As presented in Fig. $2 \mathrm{D}$ and $\mathrm{E}$, the SI/R group had a significantly lower $\mathrm{Bcl}-2 / \mathrm{Bax}$ ratio compared with the control group $(\mathrm{P}<0.05)$. When compared with the SI/R group, the adropin group exhibited a significantly higher Bcl-2/Bax ratio $(\mathrm{P}<0.05)$. Additionally, the adropin + LY294002 and adropin + PD98059 groups exhibited significantly lower $\mathrm{Bcl}-2 / \mathrm{Bax}$ ratios $(\mathrm{P}<0.05)$ compared with the adropin-only group. However, no significant differences were determined in the $\mathrm{Bcl}-2 / \mathrm{Bax}$ ratio between the adropin and adropin $+\mathrm{AG} 490$ group (Fig. 2D and E).

Effects of different doses of adropin on the inflammatory response. The inflammatory response was assessed using TNF- $\alpha$ and IL-10 expression measurements (Fig. 3A and B, respectively). TNF- $\alpha$ levels significantly increased $(\mathrm{P}<0.001)$ and IL-10 levels decreased $(\mathrm{P}<0.001)$ in the $\mathrm{SI} / \mathrm{R}$ group compared with the control group. The adropin-M and adropin-H groups exhibited significantly reduced TNF- $\alpha$ expression $(\mathrm{P}<0.001)$ and significantly increased IL-10 expression $(\mathrm{P}<0.001)$ when compared with the $\mathrm{SI} / \mathrm{R}$ group. In addition, no significant difference was determined in TNF- $\alpha$ and IL-10 expression levels between the adropin-M and adropin- $\mathrm{H}$ groups $(\mathrm{P}>0.05)$. The adropin-L group did not affect TNF- $\alpha$ or IL-10 levels compared with the control group, suggesting that moderate and high concentrations of adropin can protect the heart by alleviating the inflammatory response. 
A

Control

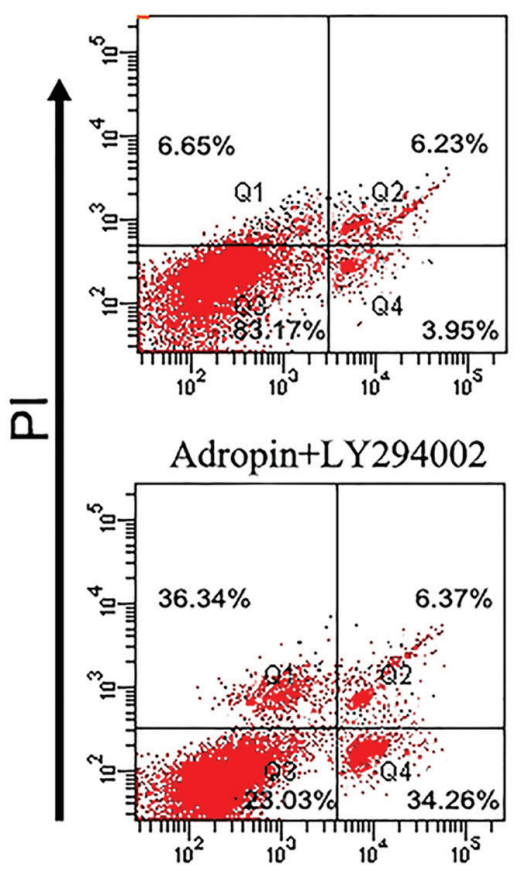

SU/R

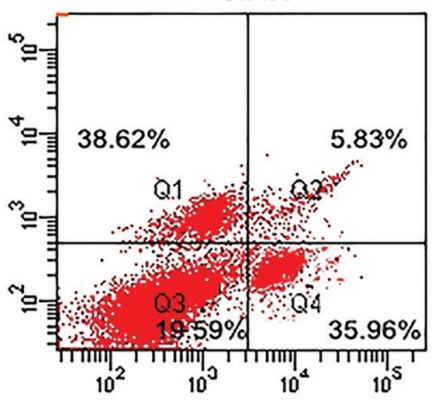

Adropin+PD98059

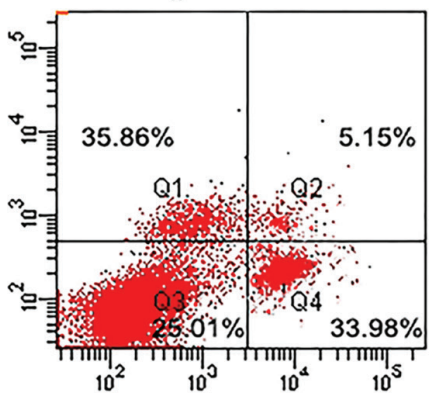

Adropin
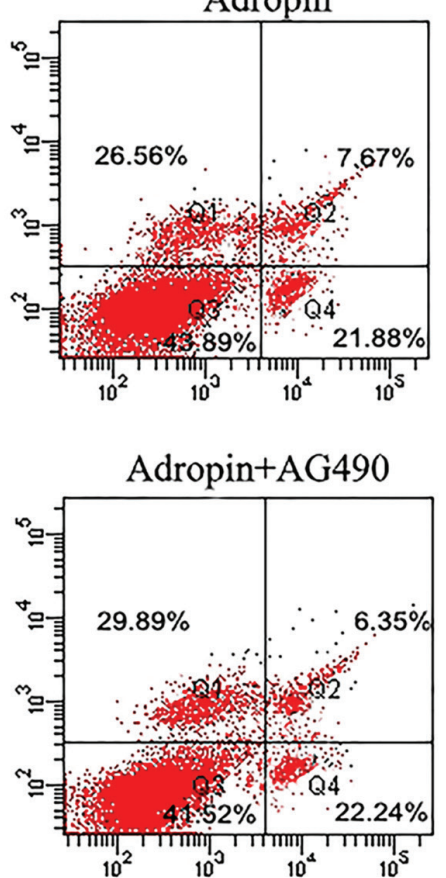

Annexin V-FITC

B

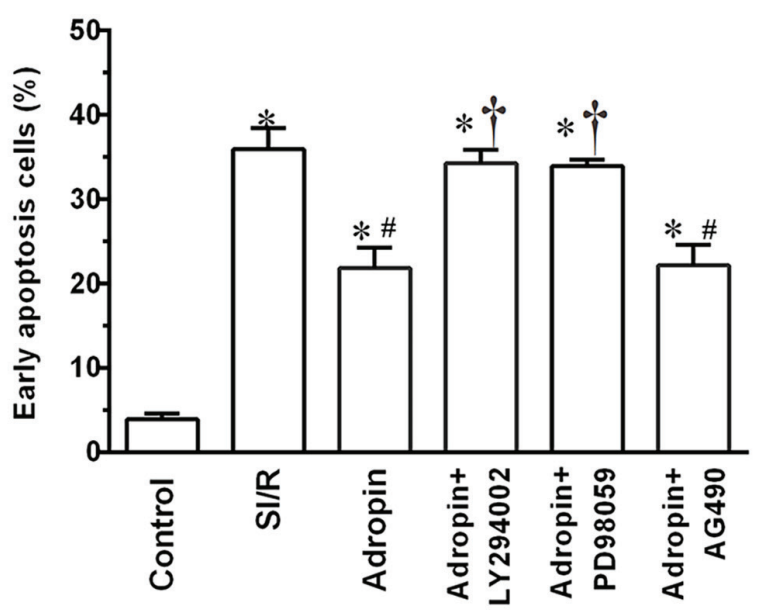

C $\overline{0} 200$

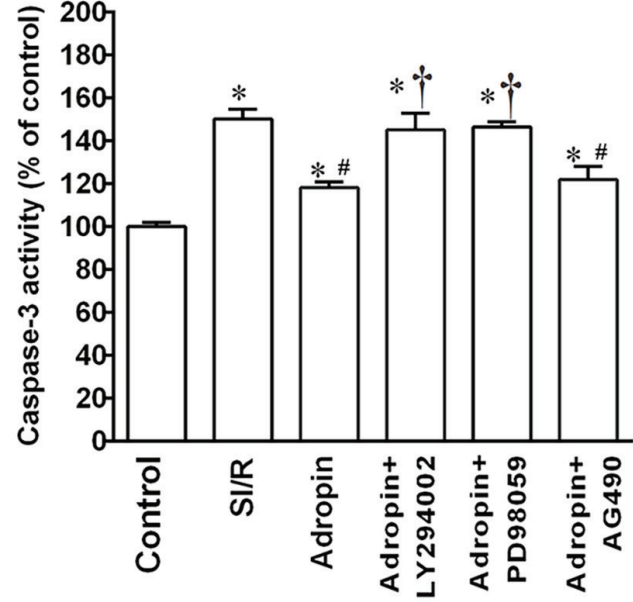

E

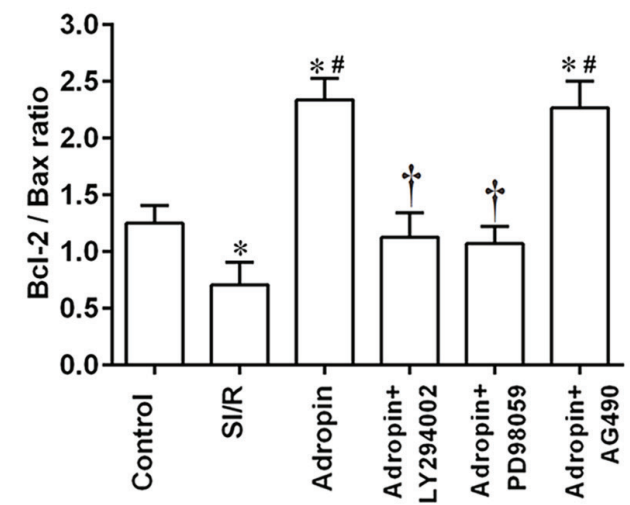

Figure 2. Effect of adropin on cell apoptosis and caspase-3 activity. (A and B) Apoptotic rates were quantified by annexin V-FITC/PI double staining cytom-

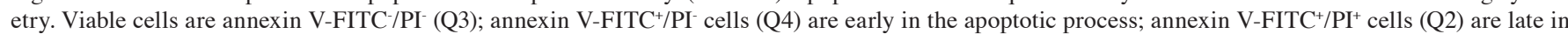
the apoptotic process; and necrotic cells are Annexin V-FITC $-\mathrm{PI}^{+}(\mathrm{Q} 1)$. (C) Caspase-3 activity analysis. " $\mathrm{P}<0.001$ vs. control; ${ }^{\prime} \mathrm{P}<0.001 \mathrm{vs}$. SI/R; ' $\mathrm{P}<0.001 \mathrm{vs}$. adropin. Results are representative of three independent experiments. (D) Western blot analysis and (E) subsequent quantification of Bcl-2 and Bax expression. Mean \pm standard deviation of relative $\mathrm{Bcl}-2 / \mathrm{Bax}$ ratios are presented. Data were normalized to loading control GAPDH. ${ }^{*} \mathrm{P}<0.05$ vs. control; ${ }^{\mathrm{P}}<0.05$ vs. SI/R; ${ }^{\dagger} \mathrm{P}<0.05$ vs. adropin. $\mathrm{PI}$, propidium iodide; SI/R, simulated ischemia/reperfusion. 
A

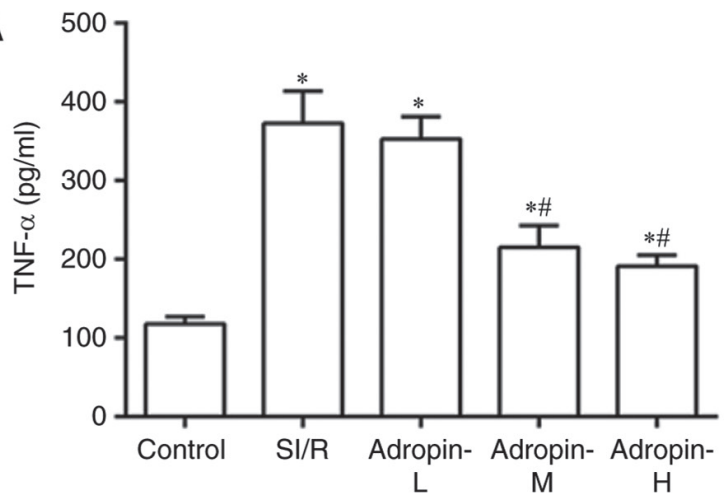

C

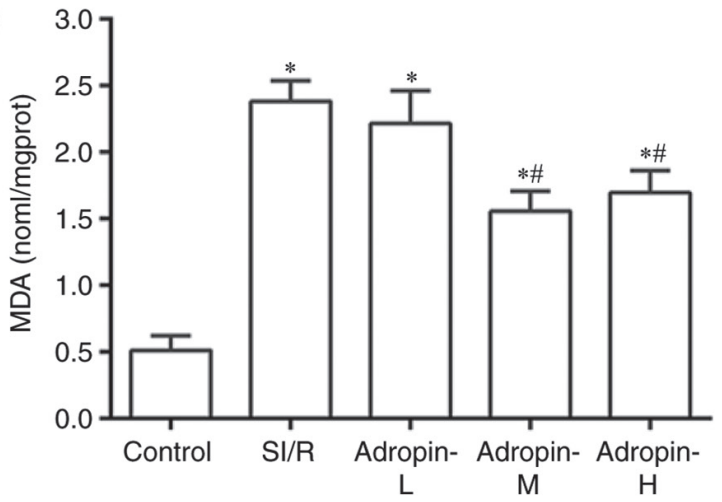

B

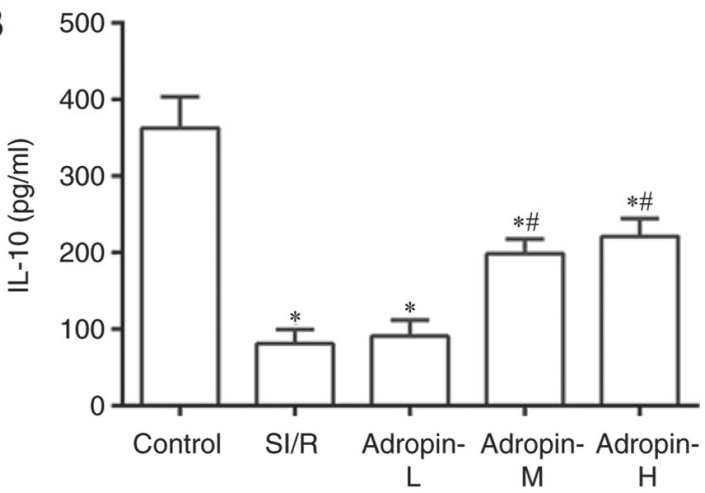

D

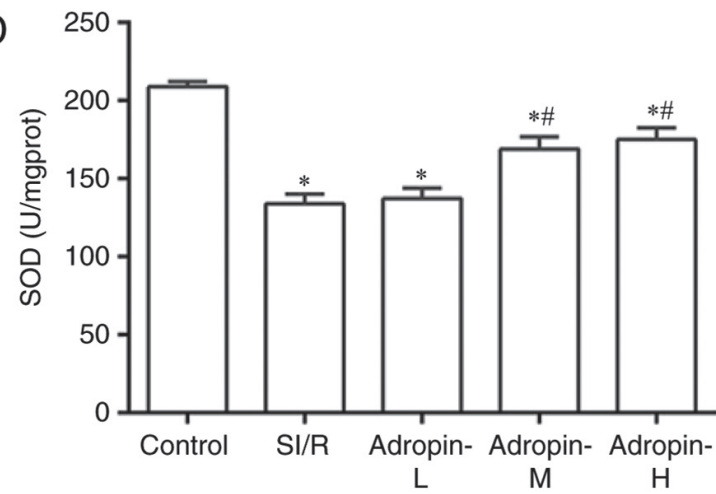

Figure 3. Effects of adropin on the inflammatory response and oxidative stress. Effect of adropin on (A) TNF- $\alpha$, (B) IL-10, (C) MDA and (D) SOD of H9c2 cells subjected to $\mathrm{SI} / \mathrm{R}$. ${ }^{*} \mathrm{P}<0.001$ vs. control and ${ }^{\#} \mathrm{P}<0.001 \mathrm{vs}$. SI/R. Results are representative of three independent experiments. TNF- $\alpha$, tumor necrosis factor $\alpha$; IL-10, interleukin 10; MDA, malondialdehyde; SOD, superoxide dismutase; SI/R, simulated ischemia/reperfusion; L, low; M, medium; H, high.

Effects of different doses of adropin on oxidative stress. Oxidative stress was examined by measuring MDA levels and SOD activity (Fig. 3C and D, respectively). MDA levels significantly increased $(\mathrm{P}<0.001)$ and SOD activity was significantly reduced in the SI/R group $(\mathrm{P}<0.001)$ compared with the control group. The adropin-M and adropin- $\mathrm{H}$ groups exhibited reduced MDA levels $(\mathrm{P}<0.001)$ and exhibited higher SOD activity $(\mathrm{P}<0.001)$ compared with the $\mathrm{SI} / \mathrm{R}$ group. The results indicated that adropin may inhibit lipid peroxide production and increase scavenging superoxide radical activity. No significant difference in MDA levels and SOD activity were determined between the adropin- $\mathrm{M}$ and adropin-H groups. Adropin-L did not reduce MDA levels or increase SOD activity when compared with the SI/R group, demonstrating the dose-dependent role of adropin in the antioxidative effect.

Reperfusion injury salvage kinase (RISK) pathway is associated with the reduction of SI/R injury by adropin. The results of the present study demonstrated that adropin inhibited myocardial injury induced by SI/R in a dose-dependent manner. The Adropin-M group (the minimum optimal concentration) was used as the Adropin group in subsequent experiments to further assess the molecular mechanisms associated with the reduction of adropin in SI/R injury.

As presented in Fig. 4A, the adropin group induced a significant elevation in $\mathrm{p}-\mathrm{Akt} / \mathrm{t}-\mathrm{Akt}$ ratio $(\mathrm{P}<0.05)$ compared with the SI/R group. LY294002 group exhibited a significantly decreased p-Akt/t-Akt ratio when compared with the adropin group $(\mathrm{P}<0.05)$. In addition, the adropin group had a significantly higher $\mathrm{p}-\mathrm{ERK} 1 / 2 / \mathrm{t}-\mathrm{ERK} 1 / 2$ ratio $(\mathrm{P}<0.05)$ compared with the SI/R group. PD98059 exhibited a significantly decreased p-ERK1/2/t-ERK1/2 ratio compared with the adropin group (Fig. 4B). Furthermore, adropin significantly increased $\mathrm{p}-\mathrm{GSK} 3 \beta / \mathrm{t}-\mathrm{GSK} 3 \beta$ ratio compared with the SI/R group $(\mathrm{P}<0.05)$, which was partially but significantly reversed by additive treatments with LY294002 $(\mathrm{P}<0.05)$ or PD98059 $(\mathrm{P}<0.05)$. However, the adropin and adropin + AG490 groups demonstrated no significant difference in the $\mathrm{p}-\mathrm{GSK} 3 \beta / \mathrm{t}-\mathrm{GSK} 3 \beta$ ratio (Fig. 4C). Compared with the control, the AG490 and adropin + AG490 groups significantly inhibited the phosphorylation levels of STAT3 $(\mathrm{P}<0.05)$. Notably, adropin and control groups exhibited no difference in the p-STAT3/t-STAT ratio (Fig. 4D).

\section{Discussion}

Adropin is a newly identified endogenous bioactive substance that serves an important role in energy metabolism. Lovren et al (6) demonstrated that adropin may directly affect endothelial cells and may possess nonmetabolic properties, including the protection of endothelial function through the RISK pathway. Adropin upregulates eNOS and increases the production of NO through the PI3K-Akt and ERK1/2 pathways. Adropin also serves a role in improving murine limb perfusion and elevating capillary density after ischemia (6). Exogenous adropin reduces insulin resistance and metabolic disorders, protects endothelial cells and attenuates organ ischemia $(6,10)$. These results indicate that adropin may also be 
A

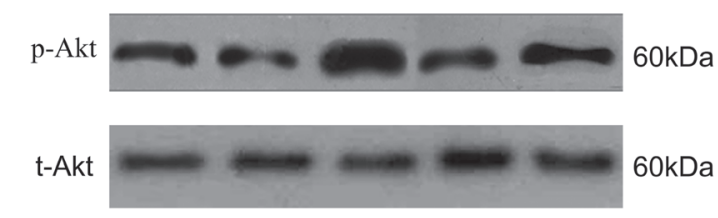

GAPDH

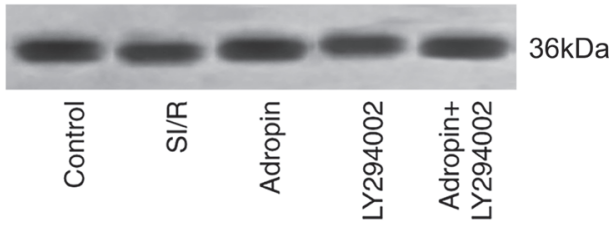

$\mathrm{B}_{\mathrm{p}-\mathrm{ERK} 1 / 2}$

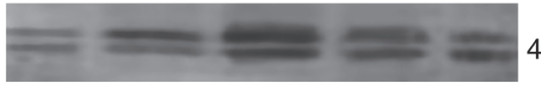

$42 / 44 \mathrm{kDa}$

t-ERK1/2

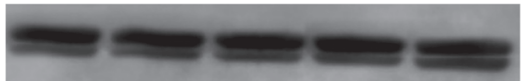

$42 / 44 \mathrm{kDa}$

GAPDH

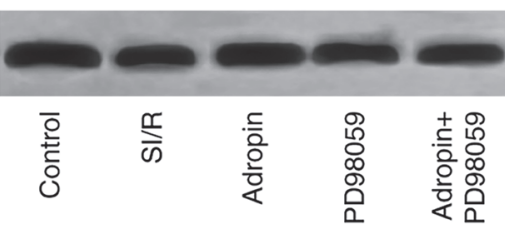

C

p-GSK3 $\beta$

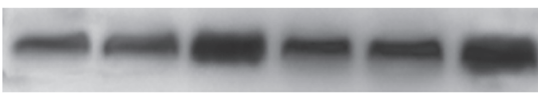

$46 \mathrm{kDa}$

t-GSK3 $\beta$

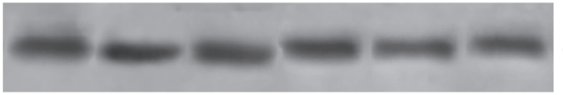
$46 \mathrm{kDa}$

GAPDH

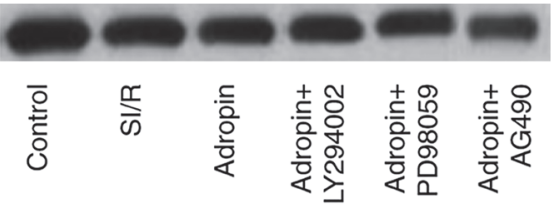

$36 \mathrm{kDa}$

D

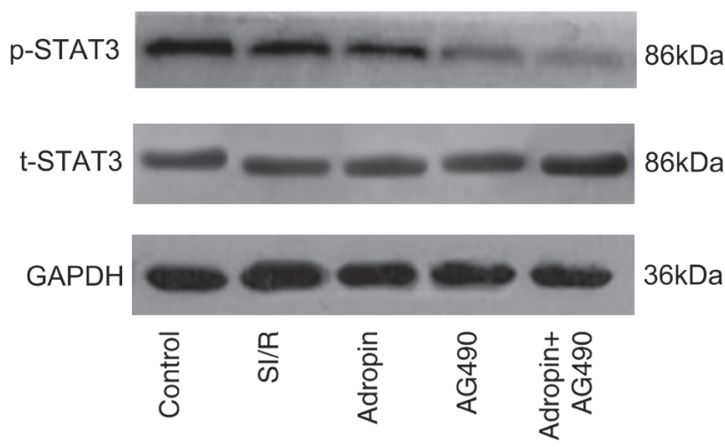

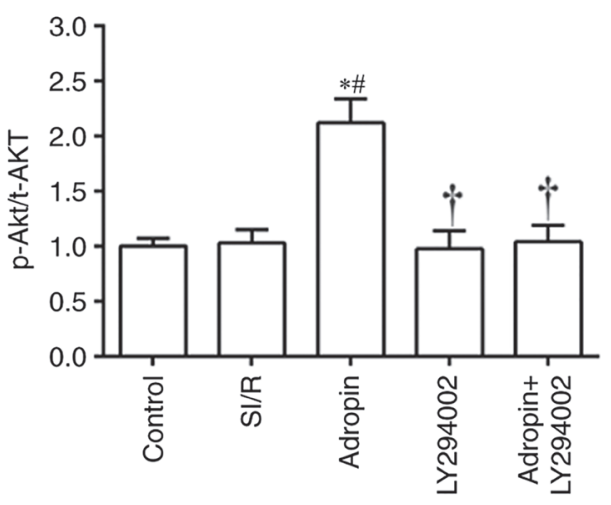
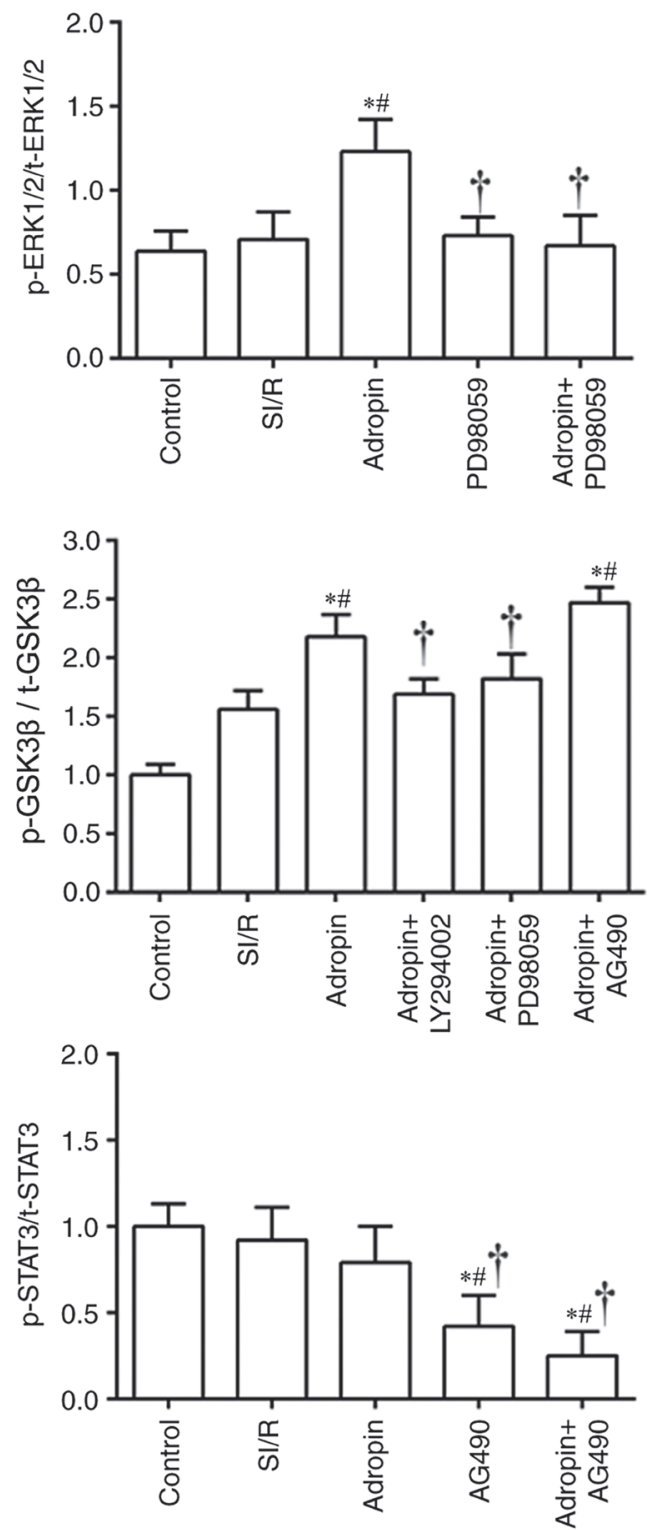

Figure 4. Effect of SI/R and adropin on the activation of the RISK pathway in H9c2 cells. Expression and subsequent quantification of (A) p-Akt and t-Akt, (B) p-ERK1/2 and t-ERK1/2, (C) p-GSK3 $\beta$ and t-GSK3 $\beta$ and (D) p-STAT3 and t-STAT3. Data are presented as the mean \pm standard deviation. Results were normalized to loading control GAPDH. ${ }^{*} \mathrm{P}<0.05$ vs. control; ${ }^{*} \mathrm{P}<0.05$ vs. SI/R. ${ }^{~ P}<0.05$ vs. adropin. P, phosphorylated; t, total; GSK3 $\beta$, glycogen synthase kinase $3 \beta ; \mathrm{SI} / \mathrm{R}$, simulated ischemia/reperfusion.

associated with ischemia reperfusion injury and may serve a cardioprotective role in MIRI.

Apoptosis is an important factor in the pathogenesis of MIRI $(14,15)$. Mitochondria serve a central role in apoptosis regulation and control cytochrome $\mathrm{C}$ release through channels formed by Bcl-2 gene family expression, which is a key mechanism that regulates apoptosis (14). The inhibition of myocardial apoptosis can prevent myocardial cell loss and delay the 
occurrence of heart failure $(16,17)$. In the present study, the results indicated that adropin treatment after hypoxia induction can inhibit hypoxia/reoxygenation-induced injury in $\mathrm{H} 9 \mathrm{c} 2$ cells. Adropin reduced the proportion of early apoptosis in myocardial cells, decreased the activity of caspase- 3 , reduced the expression of Bax gene and increased Bcl-2 gene expression. These results demonstrated that adropin can reduce SI/R injury by regulating the mitochondrial apoptosis pathway.

A number of inflammatory factors including TNF- $\alpha$, IL-1, IL-6 and IL-8 are released by myocardium subjected to ischemia-reperfusion $(18,19)$. Oxidative stress also serves an important role in myocardial injury located in the infarcted and reperfused myocardium $(20,21)$. Myocardial cells generate numerous reactive oxygen species during the ischemia-reperfusion process and increase TNF- $\alpha$ synthesis, which can lead to an increase in the apoptosis cascade reaction, the interactions between inflammatory and endothelial cells and intracellular calcium overload (22). Various novel antioxidants have been associated with renal protection through the antioxidative and antiapoptotic pathways $(23,24)$. A previous study (9) has demonstrated that adropin is negatively correlated with the inflammatory marker $\mathrm{C}$ reactive protein. In patients with severe coronary atherosclerosis, adropin serum level is low (9), which indicates that adropin possesses a potential anti-inflammatory effect. In the current study, moderate and high concentrations of adropin were indicated to reduce the inflammatory response and oxidative stress during SI/R injury. Additionally, adropin was revealed to inhibit SI/R-induced myocardial injury by reducing early myocardial apoptosis, inflammatory response and oxidative stress, and increasing myocardial cell viability.

In 2007, Yellon et al (25) proposed a new cardioprotective strategy to reduce MIRI at the early stages of reperfusion by targeting the RISK-mitochondrial permeability transition pathway (mPTP). This study revealed that ischemic or pharmacological postconditioning prior to reperfusion can activate RISK or inhibit mPTP opening to limit infarct size and reduce MIRI (25). Ischemic and pharmacological postconditioning invoke the activation of signal transduction cascades by autacoids triggers and eventually inhibit the opening of mPTP (26). However, pharmacological postconditioning performed prior to continuous reperfusion is operable in clinical practice and can avoid mechanical manipulation and associated complications (26). The activation of the RISK signaling pathway (PI3K/Akt and ERK1/2) may serve a role in cardioprotection in myocardial reperfusion and therefore, this pathway may become an important drug target (27). The activation of PI3K and its downstream target (Akt) is also associated with myocardial reperfusion injury $(28,29)$. In the ischemic myocardium, the phosphorylation of Akt can inhibit myocardial apoptosis and promote the cell survival pathway (30). Additionally, ERK1/2 is an important kinase of the RISK pathway and its activation in myocardial ischemia/reperfusion is beneficial to reduce apoptosis and to help recover cardiac function (31).

In addition to the RISK pathway, the survivor activating factor enhancement (SAFE) pathway has been revealed to be an additional pro-survival signaling pathway associated with the early reperfusion period and is composed of TNF- $\alpha$ and STAT-3 (32). mPTP is the downstream effector of the
SAFE and RISK pathways (32). mPTP may be the common final effector of cardioprotective effects exhibited by pre and postconditioning (27). Furthermore, complex crosstalk between RISK and SAFE pathways may exist.

The current study assessed whether the RISK and SAFE pathways are associated with the role of adropin in the reduction of SI/R injury in cardiomyocytes. The results demonstrated that a moderate concentration of adropin significantly increased the phosphorylation of Akt and ERK1/2 and these results are consistent with Lovren et al (6). It was also revealed that adropin can promote the phosphorylation of GSK3 $\beta$ (a prosurvival signaling pathway downstream target protein). PI3K specific inhibitor LY294002 or ERK1/2 inhibitor PD98059 also significantly inhibited the cardioprotective effects of adropin, indicating that these effects may be dependent on the PI3K/Akt and ERK1/2 pathway. The adropin treatment did not significantly increase the phosphorylation of STAT3, which is the most important target of the SAFE pathway (33). STAT3 is also the substrate of JAK2 kinase. The JAK2 kinase specific inhibitor, AG490, did not significantly inhibit the protective role of adropin in SI/R injury.

In conclusion, the results of the present study demonstrate that adropin reduces SI/R injury in $\mathrm{H} 9 \mathrm{c} 2$ myocardial cells through the RISK pathway (PI3K/Akt and ERK1/2) by activating the downstream target GSK $3 \beta$ to regulate the mitochondrial apoptosis. However, the SAFE pathway (JAK-STAT3) was not indicated to be associated with the exhibited myocardial protection. The current study may provide a potential therapeutic target for ischemia reperfusion injury and a theoretical basis for the clinical use of adropin.

Although H9c2 cells have been widely used in the study of cardiovascular disease, these studies may not accurately represent the in vivo reaction of normal myocardial cells to drug treatments. In the current study, the effects of adropin were only assessed in relation to a few inflammatory factors. However, other inflammatory factors such as leukocyte adhesion, aggregation and inflammatory stimulation signals and their receptors have not been involved. Reactive oxygen species (ROS) levels were not directly assessed and ROS scavenger was also not used. Therefore, the mechanisms underlying the changes in SOD and MDA levels observed in the current study following treatment with adropin remain to be determined.

\section{Acknowledgements}

Not applicable.

\section{Funding}

The current study was mainly supported by The National Natural Science Foundation of China (grant no. 81500352) and partially by the Natural Science Foundation of Fujian Province of China (grant no. 2016J05186), the Program for New Century Excellent Talents in Fujian Province University (grant no. 2015B021), the Medical Elite Cultivation Program of Fujian (grant no. 2015-ZQN-ZD-12), the Youth Foundation of Fujian Provincial Health and Family Planning Commission of China (grant no. 2015-1-40) and the Science Foundation for Distinguished Young Scholars of Fujian Province (grant no. 2013J06015). 


\section{Availability of data and materials}

The datasets used and/or analyzed during the current study are available from the corresponding author on reasonable request.

\section{Authors' contributions}

LW and JF performed data analysis, wrote the manuscript and contributed to the critical revision of the manuscript. LW, XY and CX conducted the experiments and statistical analysis. LC performed data analysis and contributed to the critical revision of the manuscript. All authors are the guarantors of this work and, as such, had full access to all the data in the study and take responsibility for the integrity of the data and the accuracy of the data analysis. All authors read and approved the final manuscript.

\section{Ethics approval and consent to participate}

Not applicable.

\section{Patient consent for publication}

Not applicable.

\section{Competing interests}

The authors declare that they have no competing interests.

\section{References}

1. Hausenloy DJ and Yellon DM: Myocardial ischemia-reperfusion injury: A neglected therapeutic target. J Clin Invest 123: 92-100, 2013.

2. Chandrasekar B, Smith JB and Freeman GL: Ischemia-reperfusion of rat myocardium activates nuclear factor-KappaB and induces neutrophil infiltration via lipopolysaccharide-induced CXC chemokine. Circulation 103: 2296-2302, 2001.

3. Kaminski KA, Bonda TA, Korecki J and Musial WJ: Oxidative stress and neutrophil activation-the two keystones of ischemia/reperfusion injury. Int J Cardiol 86: 41-59, 2002.

4. Eefting F, Rensing B, Wigman J, Pannekoek WJ, Liu WM, Cramer MJ, Lips DJ and Doevendans PA: Role of apoptosis in reperfusion injury. Cardiovasc Res 61: 414-426, 2004.

5. Kumar KG, Trevaskis JL, Lam DD, Sutton GM, Koza RA, Chouljenko VN, Kousoulas KG, Rogers PM, Kesterson RA, Thearle M, et al: Identification of adropin as a secreted factor linking dietary macronutrient intake with energy homeostasis and lipid metabolism. Cell Metab 8: 468-481, 2008.

6. Lovren F, Pan Y, Quan A, Singh KK, Shukla PC, Gupta M, Al-Omran M, Teoh $\mathrm{H}$ and Verma S: Adropin is a novel regulator of endothelial function. Circulation 122 (11 Suppl): S185-S192, 2010.

7. Butler AA, Tam CS, Stanhope KL, Wolfe BM,Ali MR, O'Keeffe M, St-Onge MP, Ravussin E and Havel PJ: Low circulating adropin concentrations with obesity and aging correlate with risk factors for metabolic disease and increase after gastric bypass surgery in humans. J Clin Endocrinol Metab 97: 3783-3791, 2012.

8. Celik A, Balin M, Kobat MA, Erdem K, Baydas A, Bulut M, Altas Y, Aydin S and Aydin S: Deficiency of a new protein associated with cardiac syndrome $\mathrm{X}$; called adropin. Cardiovasc Ther 31: 174-178, 2013.

9. Wu L, Fang J, Chen L, Zhao Z, Luo Y, Lin C and Fan L: Low serum adropin is associated with coronary atherosclerosis in type 2 diabetic and non-diabetic patients. Clin Chem Lab Med 52: 751-758, 2014.

10. Yang C, DeMars KM, Hawkins KE and Candelario-Jalil E: Adropin reduces paracellular permeability of rat brain endothelial cells exposed to ischemia-like conditions. Peptides 81: 29-37, 2016

11. Tanaka M, Ito H, Adachi S, Akimoto H, Nishikawa T, Kasajima T, Marumo F and Hiroe M: Hypoxia induces apoptosis with enhanced expression of Fas antigen messenger RNA in cultured neonatal rat cardiomyocytes. Circ Res 75: 426-433, 1994.
12. Singla DK, Singla RD and McDonald DE: Factors released from embryonic stem cells inhibit apoptosis in H9c2 cells through PI3K/Akt but not ERK pathway. Am J Physiol Heart Circ Physiol 295: H907-H913, 2008.

13. Ogata Y, Takahashi M, Ueno S, Takeuchi K, Okada T, Mano H, Ookawara S, Ozawa K, Berk BC, Ikeda U, et al: Antiapoptotic effect of endothelin-1 in rat cardiomyocytes in vitro. Hypertension 41: 1156-1163, 2003.

14. Scarabelli TM, Knight R, Stephanou A, Townsend P, Chen-Scarabelli C, Lawrence K, Gottlieb R, Latchman D and Narula J: Clinical implications of apoptosis in ischemic myocardium. Curr Probl Cardiol 31: 181-264, 2006.

15. Pchejetski D, Kunduzova O, Dayon A, Calise D, Seguelas MH, Leducq N, Seif I, Parini A and Cuvillier O: Oxidative stress-dependent sphingosine kinase-1 inhibition mediates monoamine oxidase A-associated cardiac cell apoptosis. Circ Res 100: 41-49, 2007.

16. Fu J, Huang H, Liu J, Pi R, Chen J and Liu P: Tanshinone IIA protects cardiac myocytes against oxidative stress-triggered damage and apoptosis. Eur J Pharmacol 568: 213-221, 2007.

17. Song JQ, Teng X, Cai Y, Tang CS and Qi YF: Activation of Akt/GSK-3beta signaling pathway is involved in intermedin(1-53) protection against myocardial apoptosis induced by ischemia/reperfusion. Apoptosis 14: 1299-1307, 2009.

18. Frangogiannis NG, Smith CW and Entman ML: The inflammatory response in myocardial infarction. Cardiovasc Res 53: 31-47, 2002.

19. Marx N, Neumann FJ, Ott I, Gawaz M, Koch W, Pinkau T and Schömig A: Induction of cytokine expression in leukocytes in acute myocardial infarction. J Am Coll Cardiol 30: 165-170, 1997.

20. Kumar D and Jugdutt BI: Apoptosis and oxidants in the heart. J Lab Clin Med 142: 288-297, 2003.

21. Kumar D, Lou H and Singal PK: Oxidative stress and apoptosis in heart dysfunction. Herz 27: 662-668, 2002.

22. Zhao ZQ: Oxidative stress-elicited myocardial apoptosis during reperfusion. Curr Opin Pharmacol 4: 159-165, 2004.

23. Liang R, Zhao Q, Jian G, Cheng D, Wang N, Zhang G and Wang F: Tanshinone IIA attenuates contrast-induced nephropathy via Nrf2 activation in rats. Cell Physiol Biochem 46: 2616-2623, 2018.

24. Kong Y, Yin J, Cheng D, Lu Z, Wang N, Wang F and Liang M: Antithrombin III attenuates AKI following acute severe pancreatitis. Shock 49: 572-579, 2018.

25. Yellon DM and Hausenloy DJ: Myocardial reperfusion injury. N Engl J Med 357: 1121-1135, 2007.

26. Ovize M, Baxter GF, Di Lisa F, Ferdinandy P, Garcia-Dorado D, Hausenloy DJ, Heusch G, Vinten-Johansen J, Yellon DM and Schulz R; Working Group of Cellular Biology of Heart of European Society of Cardiology: Postconditioning and protection from reperfusion injury: Where do we stand? Position paper from the working group of cellular biology of the heart of the European Society of Cardiology. Cardiovasc Res 87: 406-423, 2010.

27. Hausenloy DJ and Yellon DM: New directions for protecting the heart against ischaemia-reperfusion injury: Targeting the reperfusion injury salvage kinase (RISK)-pathway. Cardiovasc Res 61: 448-460, 2004.

28. Zhu M, Feng J, Lucchinetti E, Fischer G, Xu L, Pedrazzini T, Schaub MC and Zaugg M: Ischemic postconditioning protects remodeled myocardium via the PI3K-PKB/Akt reperfusion injury salvage kinase pathway. Cardiovasc Res 72: 152-162, 2006.

29. Armstrong SC: Protein kinase activation and myocardial ischemia/reperfusion injury. Cardiovasc Res 61: 427-436, 2004.

30. Mullonkal CJ and Toledo-Pereyra LH: Akt in ischemia and reperfusion. J Invest Surg 20: 195-203, 2007.

31. Jeong JJ, Ha YM, Jin YC, Lee EJ, Kim JS, Kim HJ, Seo HG, Lee JH, Kang SS, Kim YS and Chang KC: Rutin from Lonicera japonica inhibits myocardial ischemia/reperfusion-induced apoptosis in vivo and protects $\mathrm{H} 9 \mathrm{c} 2$ cells against hydrogen peroxide-mediated injury via ERK1/2 and PI3K/Akt signals in vitro. Food Chem Toxicol 47: 1569-1576, 2009.

32. Lacerda L, Somers S, Opie LH and Lecour S: Ischaemic postconditioning protects against reperfusion injury via the SAFE pathway. Cardiovasc Res 84: 201-208, 2009.

33. Lecour S: Activation of the protective survivor activating factor enhancement (SAFE) pathway against reperfusion injury: Does it go beyond the RISK pathway? J Mol Cell Cardiol 47: 32-40, 2009.

This work is licensed under a Creative Commons Attribution-NonCommercial-NoDerivatives 4.0 International (CC BY-NC-ND 4.0) License. 